\title{
Resistive switching effects on the spatial distribution of phases in metal-complex oxide interfaces
}

\author{
A. Schulman and C. Acha* \\ Laboratorio de Bajas Temperaturas, Departamento de Física, FCEyN, UBA, \\ and IFIBA (CONICET), Ciudad Universitaria, (C1428EHA) Buenos Aires, Argentina
}

\begin{abstract}
In order to determine the key parameters that control the resistive switching mechanism in metalcomplex oxides interfaces, we have studied the electrical properties of metal $/ \mathrm{YBa}_{2} \mathrm{Cu}_{3} \mathrm{O}_{7}-\delta$ (YBCO) interfaces using metals with different oxidation energy and work function ( $\mathrm{Au}, \mathrm{Pt}, \mathrm{Ag})$ deposited by sputtering on the surface of a YBCO ceramic sample. By analyzing the IV characteristics of the contact interfaces and the temperature dependence of their resistance, we inferred that ion migration may generate or cancel conducting filaments, which modify the resistance near the interface, in accordance with the predictions of a recent model.
\end{abstract}

\section{INTRODUCTION}

The resistive random access memories (RRAM) based on the non-volatile change of the resistance of metaloxide interfaces upon the application of an electric field are nowadays an interesting emerging technology $\frac{1}{\underline{1}}$ Their possible technological application as a replacement of the actual non-volatile RAM memory devices is encouraging new basic research in order to obtain a perfect control of all the parameters that influence the effect. Although many papers have been published in the last years on the resistive switching (RS) effect, observed in many metal-oxide interfaces, $\stackrel{2-11}{=}$ many aspects of the mechanism are still an open question. However, in a recent paper, Rozenberg et al .12 introduce a model that accounts for the bipolar RS phenomenon observed in transition metal oxides, highlighting the key role played by oxygen vacancies in this mechanism. Here, we analyze the temperature dependence of the RS effect in metal / ceramic YBCO interfaces with the objective to gain insight on the relevance of thermal energy on the particular characteristics of the RS change. Our results indicate that the observed temperature dependence of the resistance shift during the RS is essentially associated with the temperature dependence of the resistance of each "on" and "off" states.

\section{EXPERIMENTAL}

To study the RS effect in metal / YBCO interfaces we sputtered $4 \mathrm{Au}(\mathrm{Pt}$ or $\mathrm{Ag}$ ) electrodes (labeled 1,2,3 and 4) on one of the faces of a ceramic YBCO as it is depicted in Fig. 1. Characteristics of the textured ceramic YBCO sample can be found elsewhere $\underline{10}^{10}$

Our pulsing protocol (on electrodes 1 and 2) consisted of trains of 20,000 square pulses of amplitude $V_{- \text {pulse }}$ and $0.1 \mathrm{~ms}$ width at $1 \mathrm{kHz}$. As the pulsing generates a maximum excursion of $\sim 1 \mathrm{~K}$ on a thermometer well thermally anchored to the sample during a transient of $\sim 20 \mathrm{~s}$, we wait $60 \mathrm{~s}$ to ensure a stable temperature condition during the resistance measurement. To measure the remanent resistance after the pulsing treatment, a small bias cur-

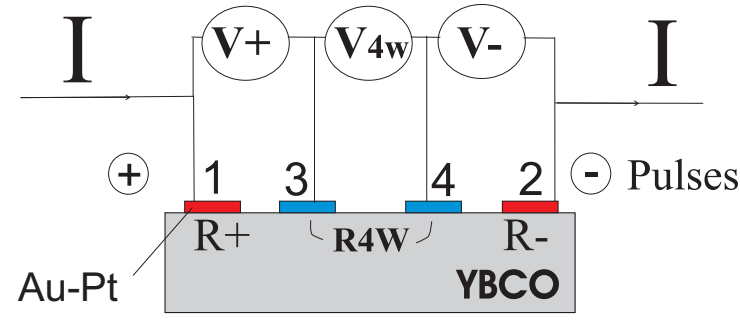

FIG. 1: (Color online) Contact configuration used to study the RS of YBCO / metal interfaces.

rent is applied to electrodes 1 and 2: by measuring the voltage drop in electrodes 1 and 3 we essentially evaluate the resistance near the interface corresponding to electrode 1 as the resistance in series coming from the bulk part of the YBCO between electrodes is negligible (confirmed by measuring the four terminal resistance $R_{4 W}$ ). The same occurs when we measure the voltage between electrodes 4 and 2 ; we essentially evaluate the resistance near the interface of electrode 2 . We arbitrarily call $R_{+}$ the former resistance $\left(V_{13} / I_{12}\right)$ as it follows the polarity of the applied pulses while the latter corresponds to $R_{-}\left(V_{42} / I_{12}\right)$. If each remanent resistance $R_{+}$or $R_{-}$ is measured after applying $V_{- \text {pulse }}$ and $V_{- \text {pulse }}$ is varied cyclically by a defined step in $\pm V_{\max }$ interval, a curve called resistance hysteresis switching loops (RHSL) can be obtained. This curve gives a clear view of the behavior of the non-volatile resistance upon the applied excitation of the pulses. Two terminals IV characteristics (1-2 electrodes) were also measured, applying a positive triangular waveform $(+10 \mathrm{~V}, 6 \mathrm{~Hz})$ to electrodes 1 and 2 in series with a calibrated $100 \Omega$ resistance, used to determine the circulating current by measuring the voltage drop between its terminals.

\section{RESULTS AND DISCUSSION}

The RS effect was observed for all the metallic electrodes we used $(\mathrm{Au}, \mathrm{Pt}, \mathrm{Ag})$, independently of their oxidation energy or their work function. The main difference observed for these metal-YBCO interfaces is asso- 
ciated with the low resistance state, where higher values were measured with decreasing the oxidation energy of the metal. Additionally, Pt-YBCO interfaces gave RHSL of lower amplitude than $\mathrm{Au}-\mathrm{YBCO}$ and $\mathrm{Ag}-\mathrm{YBCO}$, while the latter shows poor endurance. Here, we mainly show the results obtained for the Pt electrodes, which can be considered representative of the metallic interfaces studied in this work, as similar temperature effects on RS characteristics were obtained for $\mathrm{Au}$ and $\mathrm{Ag}$ electrodes.

The RHSL curves of $R_{+}$at different temperatures can be observed in Fig 2. The results for $R_{-}$, not shown here, are similar but show a complementary behavior, as when $R_{+}$switches to its low or "on" value, $R_{-}$do it to its high or "off" state $\stackrel{13}{\underline{n}}$ Decreasing the temperature clearly produces an increase of the resistance change between the two states.

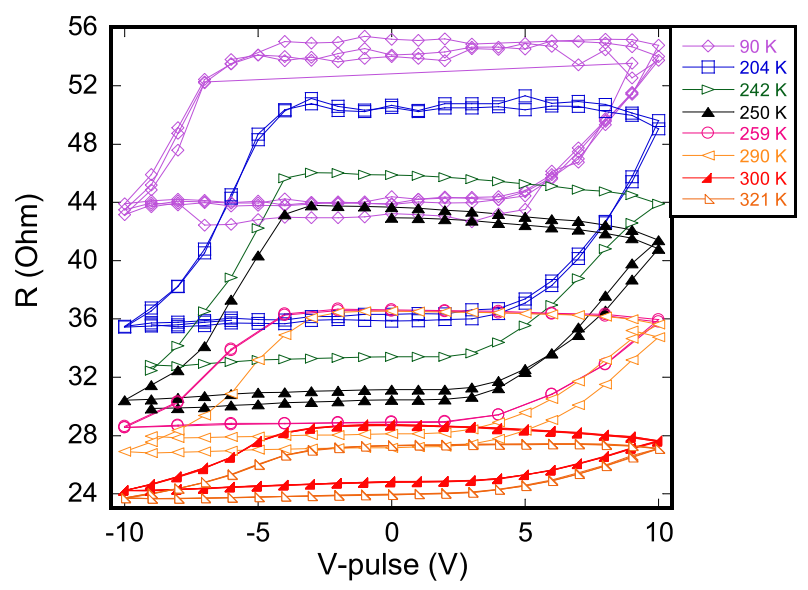

FIG. 2: (Color online) Resistance hysteresis switching loops (RHSL) at different temperatures corresponding to the nonvolatile value of $R_{+}$after applying a pulsing protocol of amplitude $V_{-p u l s e}$ (Pt-YBCO interfaces).

The temperature dependence of $R_{+}$in its "on" and "off" states (switched at room temperature) is shown in Fig 3. For both states a semiconducting-like dependence can be observed. As a consequence, their resistance difference increases with decreasing temperature. Interestingly, this temperature dependent difference is very similar to the resistance change obtained during each RHSL, as it is indicated by the lines in Fig 3 and can also be observed in Fig 4

We have previously shown 10 that pulsing can suppress and restore the superconducting state of the YBCO material in the neighborhood of the pulsed electrodes, by creating or destroying YBCO filaments, affecting the geometrical conducting factor near the interfaces. This also occurs with other oxides, where the contact resistance "copies" the temperature dependence of the bulk oxide ${ }^{8}$, probably due to the fact that the conduction near the interface is also related to conducting filaments immersed in an insulating matrix.

Within this framework, the room temperature switching between the "off" and the "on" states (or viceversa)

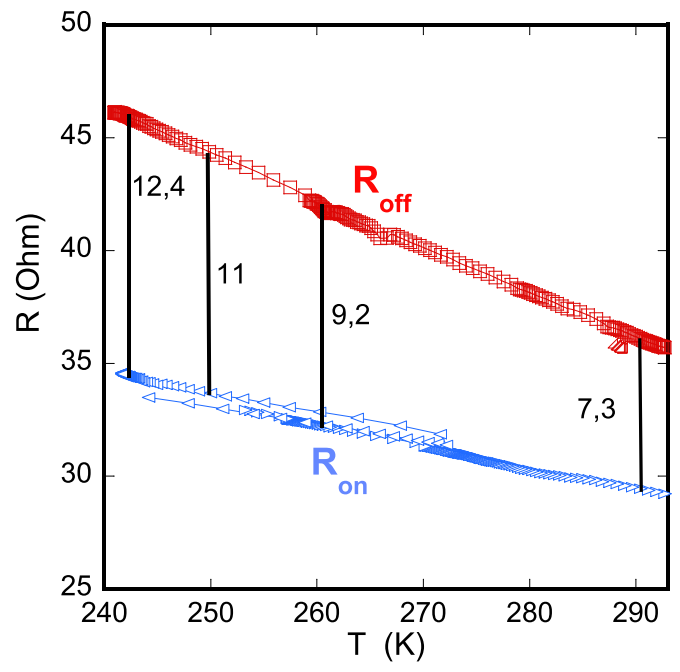

FIG. 3: (Color online) Temperature dependence of the $R_{+}$ electrode in its "on" and "off" states (Pt-YBCO interfaces). The lines indicate the shift of resistance produced by a RS at each particular temperature.

may be produced by the migration of oxygen ions that creates (or destroys) a set of well conducting YBCO filaments $\frac{12}{2}$ The coincidence of the resistance shift during the RHSL at each temperature with the resistance difference between the "on" and "off" states indicates that it is essentially the same (or an equivalent) set of filaments that is involved in the RS, independently of the temperature. In that sense, the electric field induced-spatial distribution of the conducting and insulating phases remains unchanged by varying the temperature at which the pulsing treatment is applied.

The temperature dependence of the IV characteristics of electrodes 1-2 in series, shown in Fig 5 , are consistent with this scenario. The conduction mechanism at

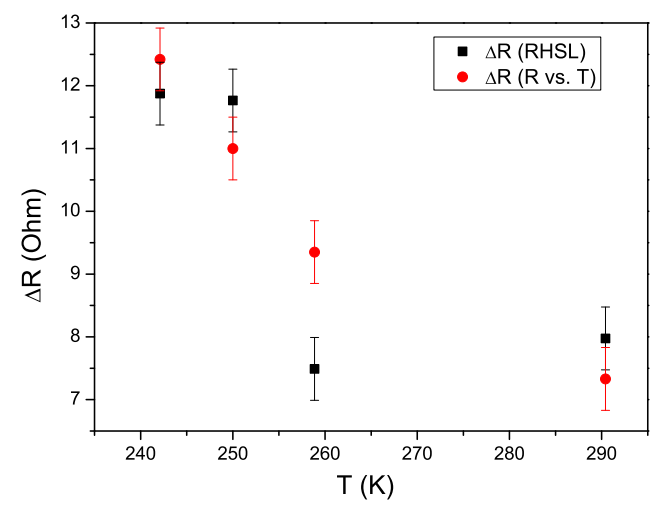

FIG. 4: (Color online) Comparison between the temperature dependent resistance difference between the "on" and "off" states and the resistance shift produced at each temperature in a RHSL (Pt-YBCO interfaces). 


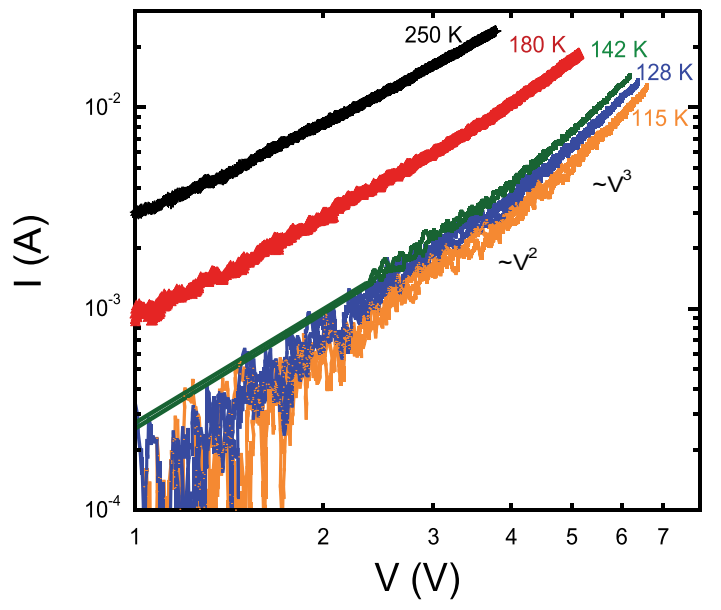

FIG. 5: (Color online) Two terminals IV characteristics at different temperatures (Au-YBCO interfaces). A power law $I \sim V^{n}$ with an increasing $n$ with increasing $\mathrm{V}$ is characteristic of a SCLC conduction mechanism.

low temperatures is of the space charge limited currents (SCLC) type.? This mechanism occurs when the conduction interface is formed by a metallic layer, capable of injecting carriers, and a semiconductor in close contact. The distribution of well oxygenated YBCO conducting zones, structurally connected to YBCO oxygen depleted surroundings may account for the observed characteristics.

\section{CONCLUSIONS}

We have studied the RS of different metal (Au, Pt, Ag) -YBCO interfaces obtaining similar effects independently of the metal used. We have shown that the temperature dependence of the resistance shift in the RHSL coincides with the difference of the resistances between the "on" and "off" states obtained during a RS at room temperature. This result indicates that although the thermal energy modifies the oxygen diffusion responsible of the $\mathrm{RS}$, the main effect of temperature is associated with the temperature dependence of the resistance of a set of conducting filaments near the interface, which can be broken or restored by the pulsing treatment, regardless of the temperature at which the process was conducted.

\section{ACKNOWLEDGMENTS}

This work was supported by CONICET Grant PIP 112-200801-00930 and UBACyT X166 (2008-2010). We acknowledge fruitful discussions with P. Levy, M. J. Rozenberg, M. J. Sánchez and R. Weht. We are indebted to D. Giménez, E. Pérez Wodtke and D. Rodríguez Melgarejo for their technical assistance.
* Corresponding author: acha@df.uba.ar

${ }^{1}$ R. F. Freitas and W. W. Wilcke, IBM J. RES. \& DEV. 52, 439 (2008).

2 A. Beck, J. G. Bednorz, C. Gerber, C. Rossel, and D. Widmer, App. Phys. Lett. 77, 139 (2000).

3 S. Q. Liu, N. J. Wu, and A. Ignatiev, App. Phys. Lett. 76, 2749 (2000).

${ }^{4}$ N. A. Tulina, A. M. Ionov, and A. N. Chaika, Physica C 366, 23 (2001).

5 S. Seo, M. Lee, D. Seo, E. Jeoung, D.-S. Suh, Y. Joung, I. Yoo, I. Hwang, S. Kim, I. Byun, et al., Appl. Phys. Lett. 85, 5655 (2004).

${ }^{6}$ R. Fors, S. Khartsev, and A. Grishin, Phys. Rev. B 71, 045305 (2005).
7 R. Waser and M. Aono, Nature Materials 6, 833 (2007).

8 M. Quintero, A. G. Leyva, and P. Levy, Appl. Phys. Lett. 86, 242102 (2005).

9 A. Sawa, Materials Today 11, 28 (2008).

10 C. Acha and M. J. Rozenberg, J. Phys.: Condens. Matter 21, 045702 (2009).

11 C. Acha, Journal of Phys. D: applied physics 44, 345301 (2011).

12 M. J. Rozenberg, M. J. Sánchez, R. Weht, C. Acha, F. Gomez-Marlasca, and P. Levy, Phys. Rev. B 81, 115101 (2010).

13 C. Acha, Physica B 404, 2746 (2009). 